\title{
The impact of two scatter correction methods on I-131 AC-SPECT images using an anthropomorphic phantom with variable sizes of thyroid remnants
}

\author{
Anastasia Hadjiconstanti* \\ Frederick Research Center, Nicosia, \\ Cyprus \\ Antonios Lontos \\ Frederick Research Center, Nicosia, \\ Cyprus and Department of \\ Mechanical Engineering, Frederick \\ University, Nicosia, Cyprus
}

\author{
Konstantinos Michael \\ Department of Mechanical \\ Engineering, Frederick University, \\ Nicosia, Cyprus and Department of \\ Medical Physics, Bank of Cyprus \\ Oncology Center, Nicosia, Cyprus \\ Savvas Frangos \\ Department of Nuclear Medicine, \\ Bank of Cyprus Oncology Center, \\ Nicosia, Cyprus
}

\author{
Theodoros Leontiou \\ Frederick Research Center, Nicosia, \\ Cyprus and Department of \\ Mechanical Engineering, Frederick \\ University, Nicosia, Cyprus
}
George Demosthenous
Department of Mechanical
Engineering, Frederick University, Nicosia, Cyprus

\author{
Maria Lyra \\ Frederick Research Center, Nicosia, \\ Cyprus
}

\begin{abstract}
Differentiated thyroid cancer treatment typically involves surgical removal of the whole or the largest part of the thyroid gland and a subsequent radioiodine therapy. It is important in diagnostic postsurgical SPECT/CT imaging to provide information on the actual presence and sizes of thyroid remnants. The aim of this work is to assess the impact of two scatter correction methods, the dual energy window (DEW) and the triple energy window (TEW), on the quality of the I-131 SPECT/CT images. Acquisitions were performed using an anthropomorphic neck-thyroid phantom with two thyroid remnants of 1.5 and $3 \mathrm{~mL}$. Activity could be injected in the remnants and the background area. For the first set of acquisitions, the counts in each thyroid remnant from the non-scatter (NSC) and scatter corrected (DEW and TEW) attenuation corrected SPECT (AC-SPECT) images for different administered activity were calculated. For the second set of acquisitions, the image quality in terms of Contrast-to-Noise (CNR), Signal-to-Noise (SNR) ratios and Noise from the NSC, DEW and TEW corrected images were calculated for different remnants-to-background activity ratios. The DEW

${ }^{*}$ Corresponding Author: Anastasia Hadjiconstanti - res.ha@frederick.ac.cy

${ }^{\dagger}$ Corresponding Author: Yiannis Parpottas - yparpottas@frederick.ac.cy

Permission to make digital or hard copies of all or part of this work for personal or classroom use is granted without fee provided that copies are not made or distributed for profit or commercial advantage and that copies bear this notice and the full citation on the first page. Copyrights for components of this work owned by others than ACM must be honored. Abstracting with credit is permitted. To copy otherwise, or republish, to post on servers or to redistribute to lists, requires prior specific permission and/or a fee. Request permissions from permissions@acm.org.

ICBBE '20, November 06-09, 2020, Kyoto, Japan

(C) 2020 Association for Computing Machinery.

ACM ISBN 978-1-4503-8822-1/20/11 . .\$15.00

https://doi.org/10.1145/3444884.3444894
\end{abstract}

\author{
Yiannis Parpottas ${ }^{\dagger}$ \\ Frederick Research Center, Nicosia, \\ Cyprus and Department of \\ Mechanical Engineering, Frederick \\ University, Nicosia, Cyprus
}

scatter correction method removed more photons than the TEW one. Even though both scatter correction methods improved image quality, especially for lower background activities and for higher volumes of remnants, this improvement is more profound when applying the TEW method. In addition, two experienced nuclear medicine physicians in a visual evaluation, without having a prior knowledge on any imaging parameters, considered that the TEW scatter corrected AC-SPECT images presented better image quality than the DEW ones. In this study, the qualitative and quantitative comparison of the two scatter correction methods indicated the effectiveness of the TEW method in diagnostic postsurgical thyroid I-131 SPECT/CT imaging.

\section{CCS CONCEPTS}

- Applied computing; • Life and medical sciences; • Health care information systems;

\section{KEYWORDS}

Differentiated thyroid cancer, SPECT/CT, Scatter Correction, Dual Energy Window, Triple Energy Window, Anthropomorphic neckthyroid phantom, Thyroid remnants

\begin{abstract}
ACM Reference Format:
Anastasia Hadjiconstanti, Konstantinos Michael, Theodoros Leontiou, Antonios Lontos, Savvas Frangos, George Demosthenous, Maria Lyra, and Yiannis Parpottas. 2020. The impact of two scatter correction methods on I-131 AC-SPECT images using an anthropomorphic phantom with variable sizes of thyroid remnants. In 2020 th International Conference on Biomedical and Bioinformatics Engineering (ICBBE '20), November 06-09, 2020, Kyoto, Japan. ACM, New York, NY, USA, 8 pages. https://doi.org/10.1145/3444884.3444894
\end{abstract}




\section{INTRODUCTION}

Differentiated thyroid cancer is deriving from the follicular epithelium and retaining basic biological characteristics of healthy thyroid tissue with a noticeable increase worldwide [1-2]. The treatment typically involves surgical removal of the whole or the largest part of the thyroid gland, total or near total thyroidectomy, and subsequent radioiodine therapy [3]. Postsurgical nuclear imaging can provide further information on the presence of thyroid remnants and/or metastasis. The diagnostic accuracy of the thyroid remnants is important for the implementation of individualized treatment for remnant ablation.

Imaging of thyroid remnant(s) can have the form of a simple pinhole scintigraphy, planar scintigraphy, single photon emission tomography (SPECT) or positron emission tomography (PET) scans. The most accurated imaging is performed with SPECT/CT after iodine 131 (I-131) administration. However, the emitted, relatively high energy, gamma rays of I-131 can scatter reducing the image contrast and degrading the image quality [4].

Several scatter correction methods have been proposed to improve the image quality [4-18]: the dual and the triple energy window scatter corrections, other energy window approaches, the deconvolution method and the spectral models. The dual energy window (DEW) method [5] subtracts a fraction of the reconstructed image using events recorded within a secondary, lower than the main photopeak, scatter window. The triple energy window (TEW) scatter correction method proposed by Ogawa [7] estimates the fraction of scattered counts in the main photopeak from upper and lower, than the main photopeak, scatter energy windows. The other energy window approaches include two or more energy windows, such as the dual photopeak window [8-11] and the multi-energy window [12]. The deconvolution method of Compton scatter uses either the measured projection data [13] or the unscatterd projection data [14]. The spectral models involve spectral fitting [15], factor analysis [16-17] or holospectral imaging [18]. In this study, the DEW and TEW scatter correction methods were compared since they are the most commonly used in practice $[4,8]$. In particular, we used the DEW scatter correction algorithm, which is the available one on the commercial Xeleris analysis workstation of the SPECT/CT modality, and our TEW developed algorithm.

The effect of scatter correction has been investigated in several studies in terms of either image quality and/or activity quantification from reconstructed SPECT images using inserts in phantoms [19-28] and mainly Tc-99m [19-22, 24-26]. There are limited studies regarding the impact of scatter correction methods on I-131 attenuation corrected SPECT (AC-SPECT) images [29-30]. In these studies, the effect of TEW scatter correction on quantitative I-131 SPECT was investigated by using phantoms of simple containers with inserts of 10-37 mL [29] and $200 \mathrm{~mL}$ [30]. More specifically, Gils et al. [29] studied the impact of various reconstruction parameters, among them the TEW scatter correction method, utilizing a body phantom with a sphere-to-background activity ratio of 10:1. Dewaraja et al. [30] assessed the quantitative accuracy of TEW scatter correction using a physical phantom with a lung insert using two different sphere-to-background activity ratios of 5:1 and 4:1.
To the best of our knowledge, the impact of both DEW and TEW scatter correction methods on the image quality of I-131 ACSPECT images utilizing an anthropomorphic phantom with thyroid remnants has not been reported. The phantom enclosed thyroid remnants of 1.5 and $3 \mathrm{~mL}$, and could simulate various remnant-tobackground activity ratios.

The quality of I-131 AC-SPECT images from these scatter correction methods was assessed by comparing images with and without scatter correction (a) for various administered activities within the thyroid remnants and (b) for various remnants-to-background activity ratios.

\section{MATERIALS AND METHODS}

\subsection{Anthropomorphic Neck-Thyroid Phantom}

The custom-made anthropomorphic neck phantom encloses humansized trachea, oesophagus and cervical spine. At the correct anatomical position, a custom-made removable thyroid-remnant section is also attached at the neck phantom. Figure 1a shows a front view of the neck-thyroid phantom (neck phantom and thyroid-remnant section) with two different sizes of thyroid remnants while Figure $1 \mathrm{~b}$ shows a coronal CT slice of the phantom, obtained with the Somaton Sensation Open (Siemens) with a slice thickness of 0.6 $\mathrm{mm}$.

The hollow cavity of the neck phantom can be filled with water $(4180 \mathrm{~mL})$ to simulate the soft tissue. Diluted radiopharmaceutical can be injected within this cavity to simulate various thyroid remnant-to-background activity ratios.

The removable thyroid-remnant section of Figure 1a encloses two hollow cavities of 1.5 and $3 \mathrm{~mL}$, at clinically relevance areas, to simulate thyroid remnants after thyroidectomy. Different removable thyroid-remnant sections with various sizes of thyroid remnants, at any clinically relevance areas, can be attached at the neck phantom. Diluted radiopharmaceuticals can be injected within the thyroid remnants.

The neck-thyroid phantom is made of a material with a density close to $1 \mathrm{~g} / \mathrm{cm} 3$. The ellipsoid hollow cavities of trachea and oesophagus are filled with air while the cylindrical hollow cavity of the cervical spine is filled with bone-equivalent material. All abovementioned parts are anatomical positioned within the phantom.

\subsection{Acquisitions}

All images were acquired with the SPECT/4-slice-CT system (Infinia Hawkeye 4, GE Healthcare) at the Department of Nuclear Medicine of the Bank of Cyprus Oncology Center. Acquisitions were performed with two High Energy General Purpose (HEGP) collimators in 1800 (H-mode) orientation. Data were acquired in 60 projections over 1800 of rotation, covering an angular range of 3600 (1800 rotation of each head). The acquisition time per projection was set to $35 \mathrm{sec}$. An $\pm 10 \%$ energy window was used for the 364 keV I-131 photopeak.

For the DEW acquisitions, the width of the scatter energy window was set to $5 \mathrm{keV}$ (304.4-309.4 keV). For the TEW acquisitions, the widths of the lower and upper energy windows were set to 5 $\mathrm{keV}$ (304.4-309.4 and 418.6-423.6 keV). All scatter windows were recorded simultaneously with the main energy window. 

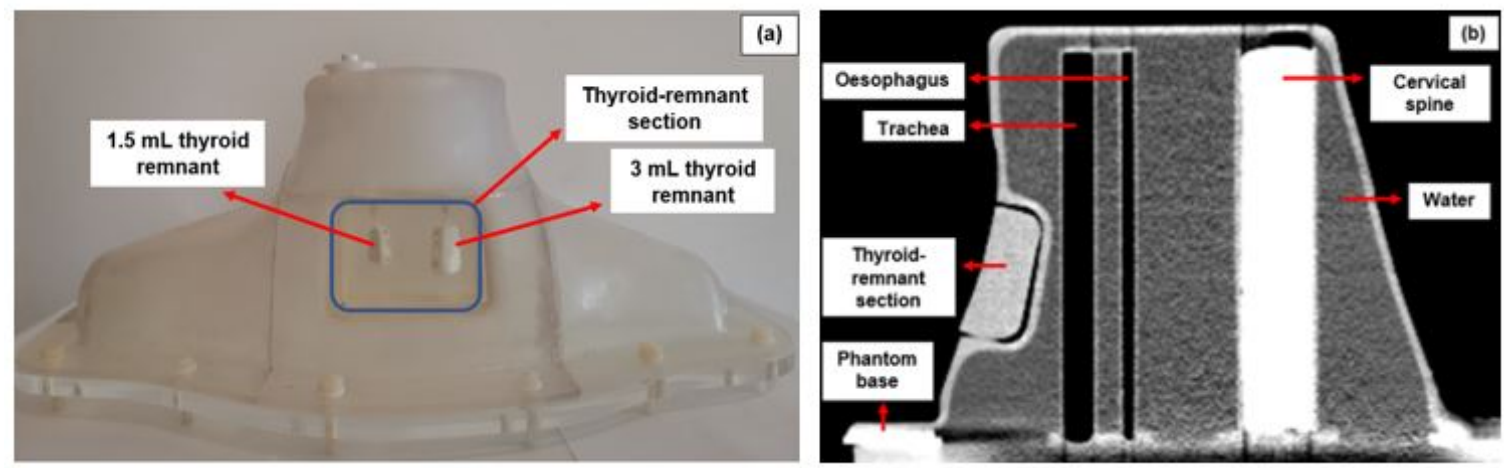

Figure 1: (a) Front view of the custom-made neck-thyroid phantom with two different sizes of thyroid remnants and (b) a coronal CT slice obtained with the Somaton Sensation Open (Siemens) with a slice thickness of $0.6 \mathrm{~mm}$

SPECT data were reconstructed using the ordered-subset expectation-maximization (OSEM) algorithm with 2 iterations and 10 subsets. The image matrix size was $128 \times 128$ with a pixel size of $4.42 \mathrm{~mm}$. A Butterworth filter (cut-off: 0.48 cycles/cm, power: 10 ) was applied to the reconstructed images.

A CT scan was also acquired at $140 \mathrm{kV}$ with $2.5 \mathrm{~mA}$. The reconstructed slice thickness was $4.42 \mathrm{~mm}$. The image matrix size was 512x512. Attenuation correction was applied to non-scattered corrected (NSC), DEW and TEW scatter corrected SPECT data.

The first set of acquisitions was performed for 5 different administered activities within the 1.5 and $3 \mathrm{~mL}$ thyroid remnants (Table 1). This range of activities can be administered for diagnostic thyroid SPECT imaging, following an almost immediate acquisition.

The second set of acquisitions was performed with 3 different background activities in the neck cavity to achieve different remnant-to-background activity ratios (5\%,10\% and 15\%). In these acquisitions, the administered activity within the remnants was $0.37 \mathrm{MBq} / \mathrm{mL}$. In diagnostic I-131 SPECT imaging, a remnant-tobackground activity ratio of $10 \%$ is considered a realistic clinical case [29].

\subsection{Scatter Correction}

The DEW scatter correction was performed by using an algorithm of the Xeleris workstation. The equation used for this scattering method is as follows:

$$
I_{D E W(p h)}=I_{N S C(p h)}-k \cdot I_{s w}
$$

where, $I_{D E W}(p h)$ is the scatter-corrected reconstructed image of the main photopeak, $I_{N C S}(p h)$ is the non-scatter corrected reconstructed image of the main photopeak which includes an unscattered component and a Compton scatter component, $I_{S W}$ is the reconstructed image of the scatter window which includes only scattered photons, and $\mathrm{k}$ is a scatter multiplier [31] which is calculated by that algorithm.

The TEW scatter correction was performed by an algorithm developed in MatLab. This software package has already been used extensively for developing algorithms related to nuclear medicine imaging [32]. The algorithm was applied to all projections prior to reconstruction. Equation 2 was used for this scatter correction method:

$$
C_{T E W(p h)}=C_{N S C(p h)}-\left(\frac{C_{\text {Lower }}}{W_{\text {Lower }}}+\frac{C_{U_{p p e r}}}{W_{U_{\text {pper }}}}\right) \cdot \frac{W_{\text {Main }}}{2}
$$

where, $C_{T E W}(p h)$ are the scatter-corrected counts in the photopeak, $C_{N S C}(p h)$ are the non-scatter corrected counts in the photopeak, $C_{\text {Lower }}$ and $C_{U \text { pper }}$ are the counts in the lower and upper scatter windows, respectively, $W_{\text {Lower }}$ and $W_{U \text { pper }}$ are the energy widths of the lower and upper scatter windows, respectively, and $W_{\text {Main }}$ is the energy width of the main photopeak window [7]. The TEW scatter-corrected data were also reconstructed by using the Xeleris workstation.

\subsection{Image Evaluation}

The total and average counts (uptake) in each thyroid remnant and in the background area were calculated. For this purpose, region-ofinterests (ROIs) were drawn in each AC-SPECT slice with observed uptake in thyroid remnants. The choice of the slices and the area of the ROIs were guided by the corresponding CT slices. As background was defined the region in the hollow cavity of the neck phantom filled with water. In each slice, the ROI of a remnant and the ROI of its corresponding background had the same area and shape. The total and average counts in a remnant and in its background area were calculated from the corresponding ROIs in the chosen slices.

For the first set of acquisitions, the total counts in each thyroid remnant from the NSC and scatter corrected (DEW and TEW) AC-SPECT images with respect to the administered activity were plotted and fitted with a linear function. For the second set of acquisitions, with background activity, the Contrast-to-Noise Ratio (CNR) and the Signal-to-Noise Ratio (SNR) values were calculated from the NSC, DEW and TEW AC-SPECT images using the average counts in each thyroid remnant and in the corresponding background areas.

The CNR is a measure to determine the image quality. A higher value of CNR may lead to an improved detectability of thyroid remnants and consequently to an improved diagnosis [29]. It is 

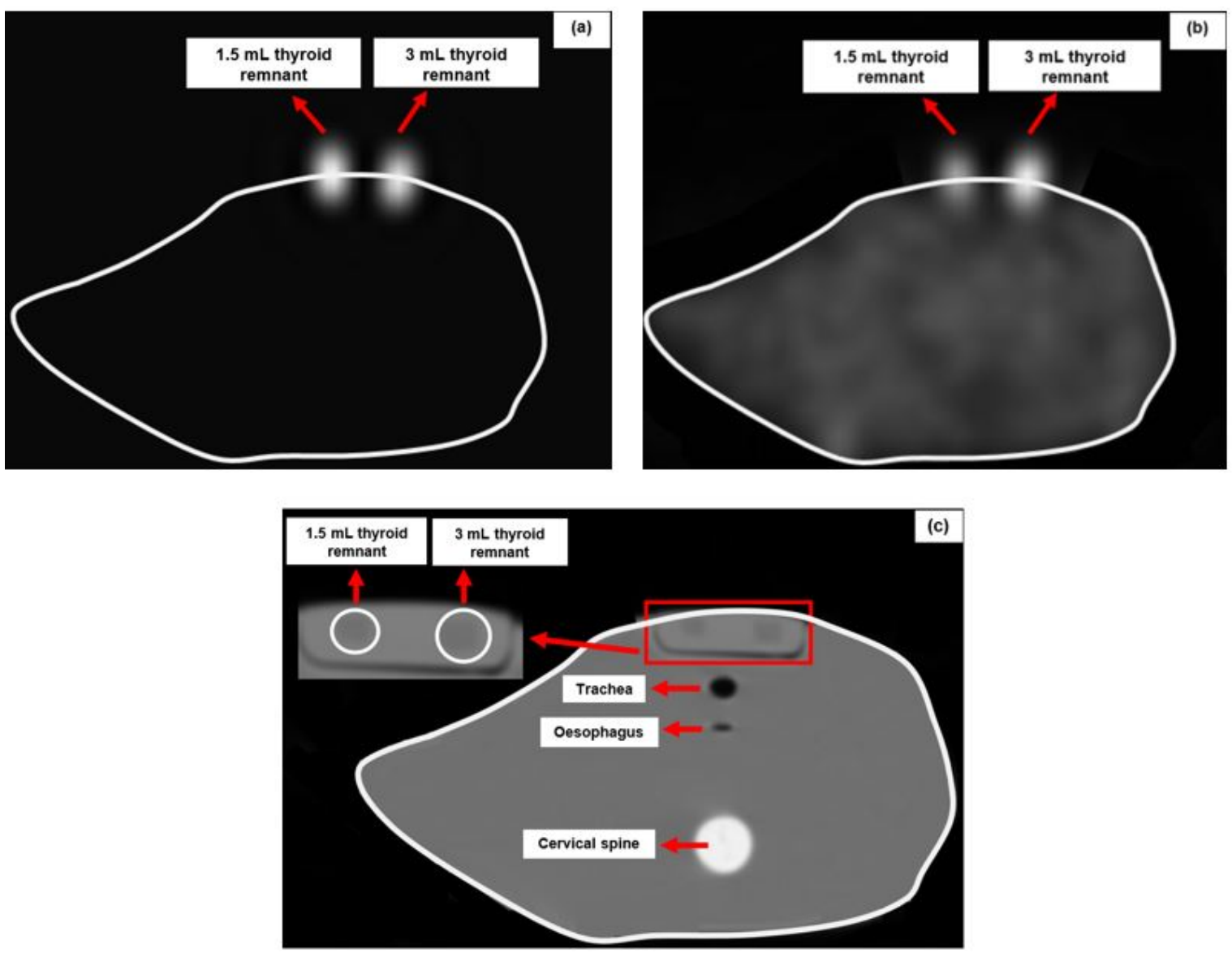

Figure 2: DEW scatter corrected AC-SPECT images: (a) without and (b) with a remnant-to-background activity ratio of 5\% and (c) the corresponding CT slice. The administered I-131 activities within the 1.5 and 3 mL thyroid remnants are written in the text. A white-color outline was drawn around the CT slice to define the borders of the phantom. The same outline was also drawn around the AC-SPECT images. The two white-color circles in the magnified region of Figure 2c indicate the corresponding thyroid remnant regions

defined as follows:

$$
C N R=\frac{C-C_{B k g}}{\sigma_{B k g}}
$$

where $C$ is the average number of counts in a remnant, $C B k g$ is the average number of counts in the corresponding background area and $\sigma \mathrm{Bkg}$ is the standard deviation of CBkg.

The SNR is another metric for image quality and subsequently for diagnosis [33- 34]. Both metrics, CNR and SNR, should be seen commentary to each other. An image may have a high SNR, that is, a large number of counts (high intensity) in the remnants, but it may also have a low CNR, that is, a large number of background counts which may lead to a low detectability of thyroid remnants. SNR is defined as follows:

$$
S N R=\frac{C}{\sigma_{B k g}}
$$

\section{RESULTS AND DISCUSSION}

Figure 2 shows DEW scatter corrected AC-SPECT images: (a) without and (b) with a remnant-to-background activity ratio of $5 \%$. For the acquisition without background activity in Figure 2a, the administered I-131 activities within the 1.5 and $3 \mathrm{~mL}$ thyroid remnants were 7.141 and $7.807 \mathrm{MBq}$, respectively, whereas the corresponding ones, for the acquisition with background activity in Figure 2b, were 0.555 and $1.110 \mathrm{MBq}$, respectively. All images in the figure are from the same slice number. The two different sizes of thyroid remnants are also shown. Figure 2c presents the corresponding CT slice. The various parts of the neck-thyroid phantom in Figure $1 \mathrm{~b}$ are also indicated in Figure 2c.

Figure 3 shows the (a) NSC, (b) DEW and (c) TEW scatter corrected AC-SPECT images for the $1.5 \mathrm{~mL}$ (left) and $3 \mathrm{~mL}$ (right) thyroid remnants (top row). The corresponding images for a 5\% remnant-to-background activity ratio are also shown in the same figure (bottom row). For the acquisitions without background activity, the administered I-131 activities within the 1.5 and $3 \mathrm{~mL}$ 

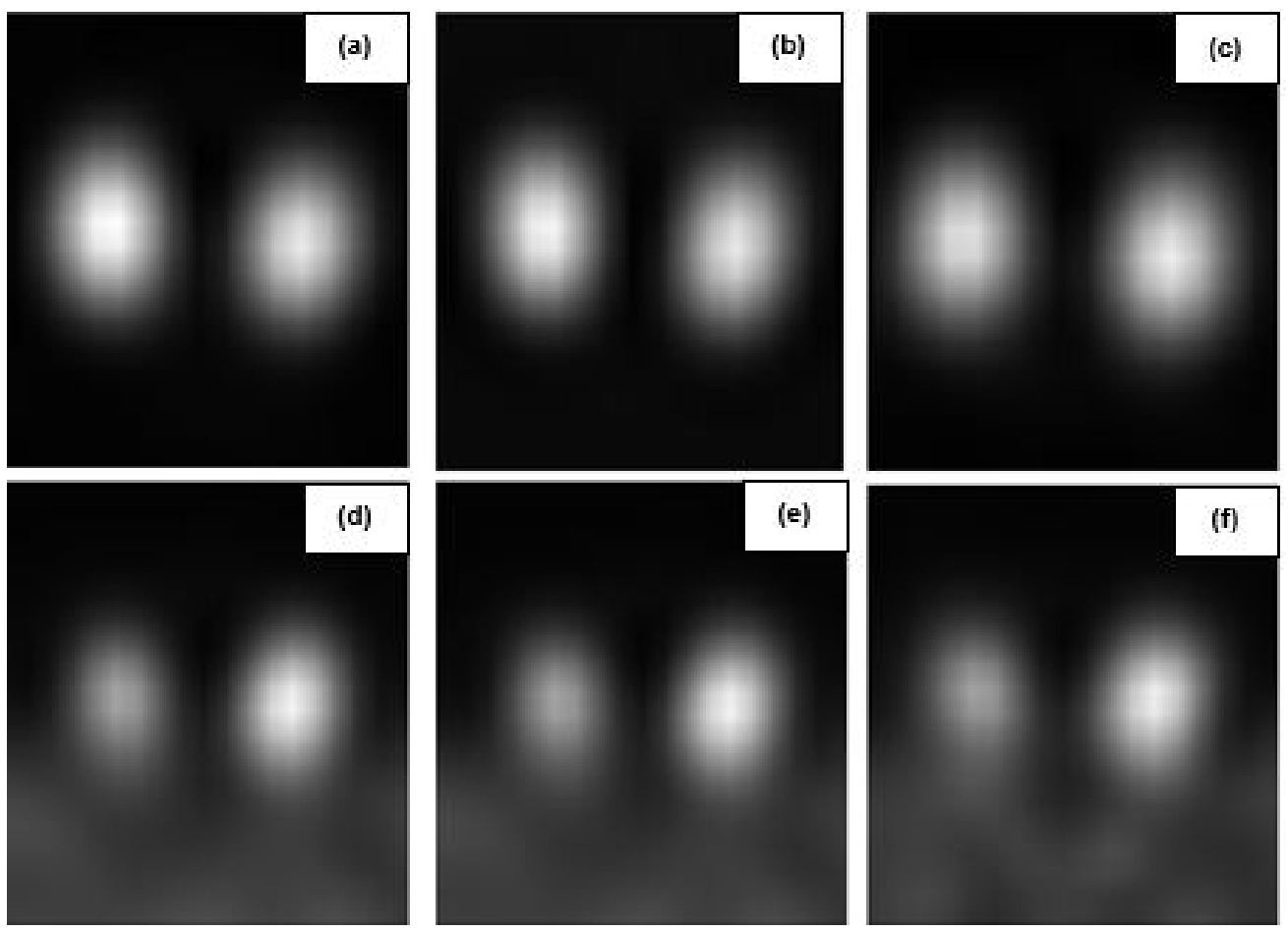

Figure 3: (a) NSC, (b) DEW and (c) TEW scatter corrected AC-SPECT images for the $1.5 \mathrm{~mL}$ (left) and $3 \mathrm{~mL}$ (right) thyroid remnants (top row), and the corresponding images with a remnant -to-background activity ratio of $5 \%$ (bottom row). The administered I-131 activities within the 1.5 and $3 \mathrm{~mL}$ thyroid remnants are written in the text

thyroid remnants were 7.141 and $7.807 \mathrm{MBq}$, respectively, whereas the corresponding ones, for the acquisitions with background activity, were 0.555 and $1.110 \mathrm{MBq}$, respectively. All images in the figure are from the same slice number. The whole set of each of the images in Figure 3 was evaluated visually by two experienced nuclear medicine physicians to decide the image (NSC or DEW or TEW image) that could draw ROIs, with more confidence, for evaluation of the volume of each remnant, which is important for therapeutic decisions. Note that the physicians had no prior knowledge of the above imaging parameters when reading the images. The physicians could not distinguish any differences among the images without background activity. However, they could be more confidence to evaluate the volume of each remnant for the TEW scatter corrected AC-SPECT images than the DEW ones, when reading the images with background activity. This is due to the resulting higher image quality of the TEW images.

The total counts in the 1.5 and $3 \mathrm{~mL}$ thyroid remnants for the different administered activity from the NSC, DEW and TEW scatter corrected AC-SPECT images are shown in Table 1. Note that, no background activity was administered for these acquisitions. Figure 4 shows the plots of the total counts in each remnant per administered activity. For each data set in the figure, the best linear fit, with zero intercept, is also presented to observe the response of the SPECT-CT modality for this range of activities. It can also be seen that the DEW method removes more photons than the TEW one. This was also found in other studies [4, 24]. In particular, for the $1.5 \mathrm{~mL}$ remnant, the NSC counts were decreased by an average of $28.4 \%$ ( $41 \%$ for the lowest and $28.3 \%$ for the highest activity) when applying the DEW method, while the corresponding NSC counts were decreased by an average of 5.9\% (6.7\% for the lowest and $11.4 \%$ for the highest activity) when applying the TEW method. For the $3 \mathrm{~mL}$ remnant, the NSC counts were decreased by an average of $28.6 \%$ ( $42.8 \%$ for the lowest and $28.2 \%$ for the highest activity) when applying the DEW method, while the corresponding NSC counts were decreased by an average of $4.6 \%$ (3.8\% for the lowest and $8.9 \%$ for the highest activity) when applying the TEW method. No any significant difference was observed $(<5 \%)$ when comparing the abovementioned decrease of counts between the two different sizes of remnants. In this range of activities, from the lowest to the highest, the abovementioned decrease of counts was changed slightly, by about $+5 \%$, when applying the TEW method, compared to the corresponding one, of about $-12 \%$, when applying the DEW method.

The CNR and SNR values for the 1.5 and the $3 \mathrm{~mL}$ thyroid remnants from the NSC, DEW and TEW scatter corrected AC-SPECT images with different remnant-to-background activity ratios (\% $\mathrm{Bkg}$ ) were calculated and are shown in Table 2. Note that, the negative values of CNR for the $15 \% \mathrm{Bkg}$ indicate increased counts in the background area compared to the corresponding counts in the remnant. Figure 5 presents the plots of the CNR and the SNR values for the different remnant-to-background activity ratios, for each thyroid remnant. 
Table 1: The total counts in the 1.5 and $3 \mathrm{~mL}$ thyroid remnants for the different administered activity from the NSC, DEW and TEW scatter corrected AC-SPECT images

\begin{tabular}{llllllll}
\hline & \multicolumn{3}{c}{ Counts (1.5 mL Thyroid Remnant) } & \multicolumn{3}{c}{ Counts (3 mL Thyroid Remnant) } \\
\hline Activity (MBq) & NSC & DEW & TEW & Activity (MBq) & NSC & DEW & TEW \\
\hline 0.962 & 47012 & 27715 & 43858 & 0.888 & 40235 & 23016 & 38709 \\
7.141 & 350508 & 259766 & 340139 & 7.807 & 343017 & 244736 & 317506 \\
13.135 & 565582 & 454927 & 554270 & 14.319 & 543789 & 437997 & 532913 \\
23.902 & 112085 & 814330 & 973337 & 26.048 & 110452 & 838409 & 1022869 \\
43.771 & 195963 & 1404925 & 1736730 & 47.804 & 193746 & 1391143 & 1764627 \\
\hline
\end{tabular}

Table 2: CNR and SNR values for the $1.5 \mathrm{~mL}$ and the $3 \mathrm{~mL}$ thyroid remnants from the NSC, DEW and TEW scatter corrected AC-SPECT images with different remnant-to-background activity ratios (\% $\mathrm{Bkg})$

\begin{tabular}{|c|c|c|c|c|c|c|}
\hline \multicolumn{7}{|c|}{$1.5 \mathrm{~mL}$ Thyroid Remnant } \\
\hline \multirow[t]{2}{*}{$\% \mathrm{Bkg}$} & \multicolumn{2}{|c|}{ NSC } & \multicolumn{2}{|c|}{ DEW } & \multicolumn{2}{|c|}{ TEW } \\
\hline & $\mathrm{CNR}$ & SNR & $\mathrm{CNR}$ & SNR & $\mathrm{CNR}$ & SNR \\
\hline 5 & 3.21 & 7.97 & 3.37 & 8.18 & 5.24 & 11.60 \\
\hline 10 & 0.54 & 4.10 & 0.67 & 4.12 & 1.34 & 4.32 \\
\hline 15 & -1.70 & 3.49 & -1.06 & 4.01 & -1.00 & 4.26 \\
\hline \multicolumn{7}{|c|}{3 mL Thyroid Remnant } \\
\hline 5 & 7.60 & 12.35 & 7.93 & 12.75 & 10.88 & 17.24 \\
\hline 10 & 2.31 & 5.87 & 2.44 & 5.90 & 3.29 & 6.26 \\
\hline 15 & 0.55 & 5.71 & 0.59 & 5.76 & 0.65 & 5.90 \\
\hline
\end{tabular}

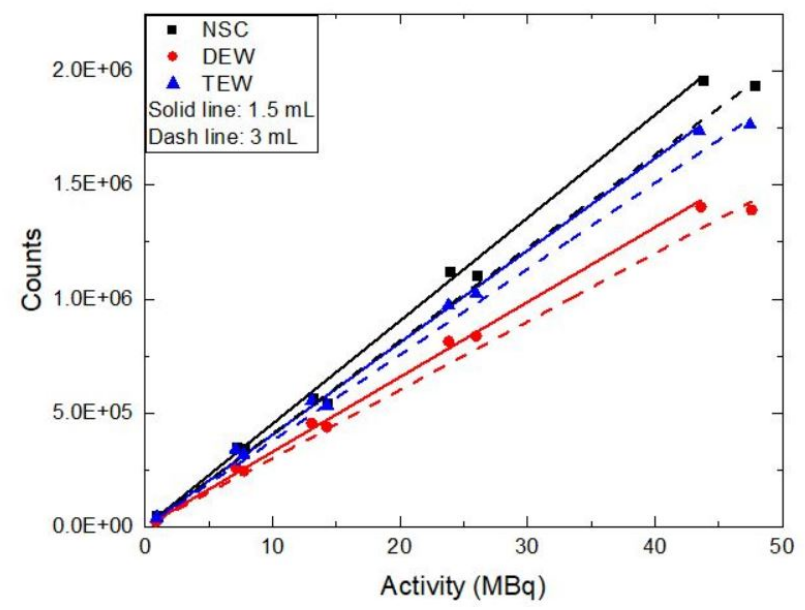

Figure 4: The total counts in the 1.5 and $3 \mathrm{~mL}$ thyroid remnants per administered activity from the NSC, DEW and TEW scatter corrected AC-SPECT images

When applying any of these scattering correction methods, both CNR and SNR values for both remnants were increased, thus improving the image quality. This is in agreement with other studies [4, 19-24]. The abovementioned increase is more profound when using the TEW method. In all cases, CNR and SNR values, for both remnants, were decreased with higher remnant-to-background activity ratios, as expected, and both scattering methods may be considered
Table 3: Noise in the NSC, DEW and TEW scatter corrected AC-SPECT images for each remnant-to-background activity ratio $(\% \mathrm{Bkg})$

\begin{tabular}{llll}
\hline$\%$ Bkg & NSC & DEW & TEW \\
\hline 5 & 38 & 36 & 24 \\
10 & 80 & 78 & 62 \\
15 & 89 & 87 & 52 \\
\hline
\end{tabular}

not so much effective for the $15 \%$ Bkg when using I-131. However, Dewaraja et al. [30] investigated the TEW scatter correction on I-131 AC-SPECT images using two different sphere-to-background activity ratios (5:1 and 4:1) and concluded that scatter correction results in a better accuracy over the examined backgrounds.

In addition, the CNR and SNR values for the $3 \mathrm{~mL}$ remnant were higher than the corresponding ones for the $1.5 \mathrm{~mL}$ remnant. The same was concluded in other studies [24, 26] with Tc-99m, using phantoms with lesions of slightly higher volumes. It is possible that the CNR and SNR values for the smallest remnant may be improved when applying, beforehand, a correction for the partial volume effect (PVE).

The noise in the NSC, DEW and TEW scatter corrected ACSPECT images for each \% Bkg were calculated and presented in Table 3. It is defined as the standard deviation of the total counts in a background area $(\sigma \mathrm{Bkg})$ [35]. In all cases, the noise was reduced for lower \% Bkg, as expected. The DEW method slightly reduced the noise while the TEW was more efficient in reducing the noise. 

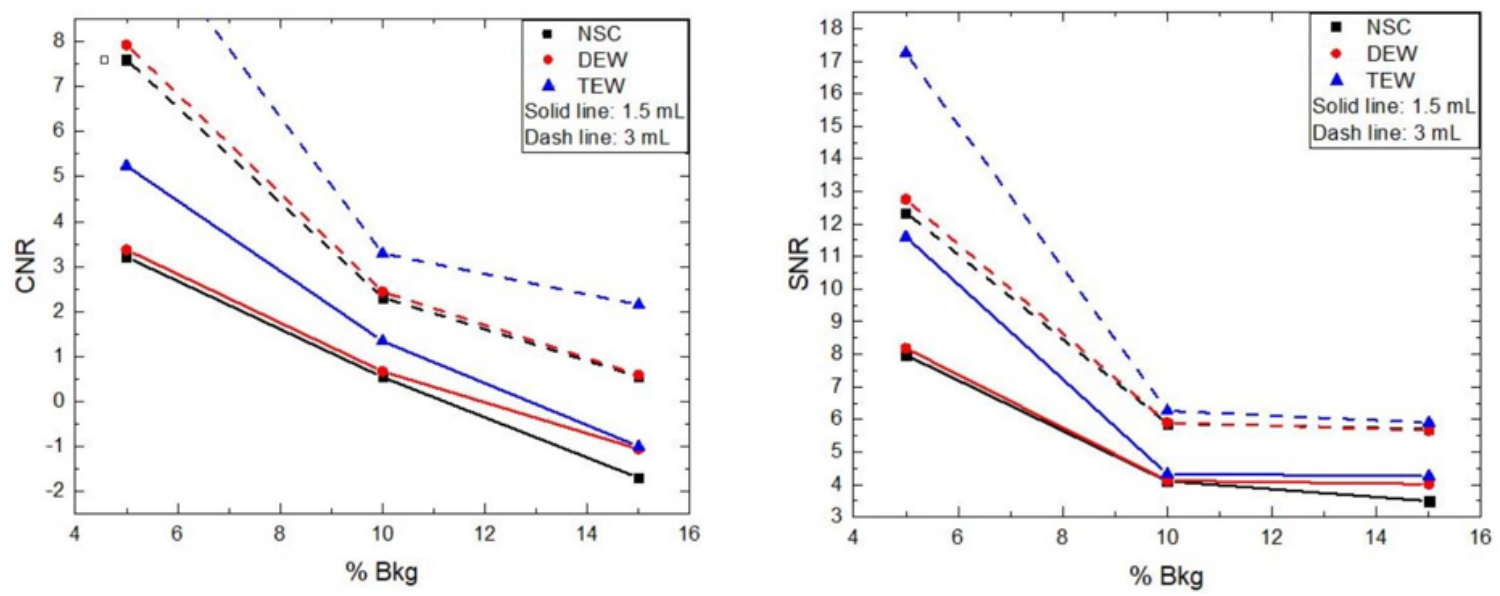

Figure 5: (Left) The CNR and (right) the SNR values for the three different thyroid remnants-to-background activity ratios (\%Bkg)

In this study, for the first time, the DEW and TEW scatter correction methods were directly compared in terms of image quality, and in particular in terms of CNR, SNR and Noise values, from diagnostic thyroid I-131 AC-SPECT images using an anthropomorphic neck-thyroid phantom with two different small sizes of thyroid remnants and three different $\% \mathrm{Bkg}$. Also, the impact of these two scatter correction methods on image quality was determined by two experienced physicians. Only a previous study compared the CNR values derived from these two scatter correction methods on Tc-99m AC-SPECT images using a liver phantom with spheres [24]. Another two previous studies only investigated the TEW method on I-131 AC-SPECT images but in terms of other parameters using phantoms with higher volume of inserts and with different background activity ratios [29-30].

The main limitation was the PVE that should be corrected for small sizes of thyroid remnants. However, this qualitative and quantitative comparison could evaluate the effectiveness of each scatter correction method in diagnostic thyroid I-131 SPECT imaging after thyroidectomy.

\section{CONCLUSION}

In this study, the performance of the commercial DEW scatter correction algorithm used at the Department of Nuclear Medicine of the Bank of Cyprus Oncology Center and that of a developed TEW scatter correction algorithm was investigated on AC-SPECT imaging for different I-131 administered activities and for different remnant-to-background activity ratios. For this purpose, an anthropomorphic neck-thyroid phantom with 1.5 and $3 \mathrm{~mL}$ sizes of thyroid remnants was utilized. The administered activities in the remnants and in the background area as well as the sizes of remnants can be considered similar to diagnostic I-131 postsurgical thyroid SPECT/CT imaging clinical scenarios.

Two experienced nuclear medicine physicians considered that the TEW scatter corrected AC-SPECT images presented better image quality than the DEW ones when a background activity was administered in the phantom and, consequently, they could be confident to evaluate the volume of the thyroid remnants.

For these administered activities, the DEW scatter correction method removed more photons than the TEW one. This photon removal changed more rapidly with the increase of the administered activity when applying the DEW than the TEW method.

For the used remnant-to-background activity ratios, both scatter correction methods improved the image quality. This improvement was more profound for the lower background activity ratios and for the remnant of higher volume. However, when comparing the two scattering methods, the TEW method improved more the image quality. In addition, the TEW method could reduce the noise in a higher extent than the DEW one.

This qualitative and quantitative comparison among the DEW and TEW scatter correction methods indicates the effectiveness of the TEW one in diagnostic postsurgical thyroid I-131 SPECT/CT imaging.

Future related studies will involve evaluation of the DEW and TEW scatter correction methods on partial-volume corrected I-131 AC-SPECT images. Furthermore, these investigations will also be performed for diagnostic thyroid I-123 AC-SPECT imaging.

\section{ACKNOWLEDGMENTS}

We greatly acknowledge Associate Professor Dr. Med. Ioannis Iakovou, Head of the 2nd Nuclear Medicine Department of the Aristotle University AHEPA Academic Hospital of Thessaloniki for visual evaluation of the images. This work was co-funded by the European Regional Development Fund and the Republic of Cyprus through the Research and Innovation Foundation (Project: EXCELLENCE/1216/0088).

\section{REFERENCES}

[1] Luster, M., Clarke, S. E., Dietlein, M., Lassmann, M., Lind, P., Oyen, W. J. G., Tennvall, J. and Bombardieri, E. 2008. Guidelines for radioiodine therapy of differentiated thyroid cancer. Eur. J. Nucl. Med. Mol. Imaging 35, 1941-59.

[2] Hodgson, N. C., Button, J. and Solorzano, C. C. 2004. Thyroid cancer: is the incidence still increasing? Ann. Surg. Oncol. 11, 1093-7. 
[3] Frangos, S., Iakovou, P. I., Marlowe, R. J., Eftychiou, N. Patsali, L., Vanezi, A., Savva, A., Mpalaris, V. and Giannoula, E. I. 2015. Difficulties in deciding whether to ablate patients with putatively "low-intermediate-risk" differentiated thyroid carcinoma: do guidelines mainly apply in the centres that produce them? Results of a retrospective, two-centre quality assurance study. Eur. J. Nucl. Med. Mol. Imaging 42, 2045-55.

[4] Hutton, B. F., Buvat, I. and Beekman, F. J. 2011. Review and current status of SPECT scatter correction. Phys. Med. Biol. 56, R85-112.

[5] Jaszczak, R. J., Greer, K. L., Floyd, C. E., Jr, Harris C.C., and Coleman, R. E. 1984 Improved SPECT quantification using compensation for scattered photons. J Nucl. Med. 25, 893-900.

[6] Jaszczak, R. J, Greer, K. L., Floyd, C. E. and Coleman, R. E. 1985. Scatter compensation techniques for SPECT. IEEE Trans. Nucl. Sci. 32, 786-93.

[7] Ogawa, K., Harata, Y., Ichihara, T. Kubo A. and Hashimoto, S. 1991. A practical method for position dependent Compton-scatter correction in single photon emission CT. IEEE Trans. Med. Imaging 10, 408-12.

[8] Buvat, I., Rogriguez-Villafuerte, M., Todd-Pokropek, A., Benali, H. and Paola, R D. 1995. Comparative assessment of nine scatter correction methods based on the spectral analysis using Monte Carlo simulations. J. Nucl. Med. 36, 1476-88.

[9] Logan, K. W. and McFarland, W. D. 1992. Single photon scatter compensation by photopeak energy distribution analysis. IEEE Trans. Med. Imaging 11, 161-4.

[10] King, M. A., Hademenos, G. J. and Glick, S. J. 1992. A dual-photopeak window method for scatter correction. J. Nucl. Med. 33, 605-12.

[11] Pretorius, P. H., van Rensburg, A. J., van Aswegen, A., Lötter, M. G., Serfontein, D. E. and Herbst, C. R. 1993. The channel ratio method of scatter correction for radionuclide image quantitation. J. Nucl. Med. 34, 330-5.

[12] Vija, H., Kaplan, M. S. and Haynor, D. R. 1999. Simultaneous estimation of SPECT activity and attenuation distributions from measured phantom data using a differential attenuation method. IEEE NSS MIC Conf. Records 2, 884-8.

[13] Axelsson, B., Msaki, P. and Israelsson, A. 1984. Subtraction of Compton-scattered photons in single photon emission computerized tomography. J. Nucl. Med. 25, 490-4.

[14] Floyd, C. E., Jr, Jaszczak, R. J., Greer, K. L. and Coleman, R. E. 1985. Deconvolution of Compton scatter in SPECT. J. Nucl. Med. 26, 403-8.

[15] Koral, K. F., Wang, X., Rogers, W. L., Clinthorne, N. H. and Wang, X. 1988. SPECT Compton scattering correction by analysis of energy spectra. J. Nucl. Med. 29, 195-202.

[16] Mas, J., Hannequin, P., Ben Younes, R. Bellaton, B. and Bidet, R. 1990. Scatter cor rection in planar imaging and SPECT by constrained factor analysis of dynamic structures (FADS). Phys. Med. Biol. 35, 1451-65.

[17] Buvat, I. Benali, H. Frouin, F. Bazin, J. P. and Di Paola, R. 1993. Target apex-seeking in factor analysis of medical image sequences. Phys. Med. Biol. 38, 123-38.

[18] Gagnon, D., Laperrière, L., Pouliot, N., Arsenault, A., Grégoire, J. and Dupras, G. 1990. Implementation of holospectral imaging on gamma camera. J. Nucl. Med. 31, 758-9.

[19] Beekman, F. J., Kamphuis, C. and Frey, E. C. 1997. Scatter compensation methods in 3D iterative SPECT reconstruction: A simulation study. Phys. Med. Biol. 42,
1619-32

[20] Buvat, I., De Sousa, M. C., Di Paola, M., Richard, M., Lumbroso, J. and Aubert, B. 1998. Impact of scatter correction in planar scintimammography: a phantom study. J. Nucl. Med. 39, 1590-96.

[21] Frey, E. C., Gilland, K. L. and Tsui, B. M. 2002. Application of task - based measures of image quality to optimization and evaluation of three-dimensional reconstruction-based compensation methods in myocardial perfusion SPECT. IEEE Trans. Med. Imaging 21, 1040-50.

[22] Xiao, J., de Wit, T. C., Staelens, S. G. and Beekman, F. J. 2006. Evaluation of 3D Monte Carlo-based scatter correction for $99 \mathrm{mTc}$ cardiac perfusion SPECT. J. Nucl. Med. 47, 1662-9.

[23] Xiao, J., de Wit, T. C., Zbijewski, W., Staelens, S. G. and Beekman, F. 2007. Evaluation of 3D Monte Carlo-based scatter correction for 201Tl cardiac perfusion SPECT. J. Nucl. Med. 48, 637-44.

[24] Perisinakis, K., Karkavitsas, N., Damilakis, J. and Gourtsoyiannis, N. 1998. Effect of dual and triple energy window scatter correction methods on image quality in liver scintigraphy. Nuklearmedizin 37, 239-44

[25] Bong, J-K., Son, H-K., Lee, J. D., and Kim, H-J. 2005. Improved scatter correction for SPECT images: A Monte Carlo study. IEEE Trans. Nucl. Sci. 52, 1263-70.

[26] Noori-Asl, M., Sadremomtaz, A., and Bitarafan-Rajabi, A. 2014. Evaluation of three scatter correction methods based on estimation of photopeak scatter spectrum in SPECT imaging: A simulation study. Phys. Med. 30, 947-953.

[27] Robinson, A. P., Tipping, J., Cullen, D. M. and Hamilton, D. 2016. The influence of triple energy window scatter correction on activity quantification for $177 \mathrm{Lu}$ molecular radiotherapy. Phys. Med. Biol. 61, 5107-27.

[28] Lyra, M. E., Lagopati, N. and Sotiropoulos, M. 2010. The impact of scattering and peak spectrum of I-123 in scintigraphy by I-123 MIBG. In A. Charalambopoulos, D.I. Fotiadis ed. Advanced topics in scattering theory and biomedical engineering, World Scientific Publishing Company, 79-88.

[29] Van Gils, C. A. J., Beijst, C. R., Van Rooij, R. and de Jong H. W. A. M. 2016. Impact of reconstruction parameters on quantitative I-131 SPECT. Phys. Med. Biol. 61, 5166-82.

[30] Dewaraja, Y., Li, J. and Koral, K. 1998. Quantitative 131I SPECT with triple energy window Compton scatter correction. IEEE Trans. Nucl. Sci. 45, 3109-14.

[31] Koral, K. F., Swailem, F. M., Buchbinder, S., Clinthorne, N. H., Rogers, W. L. and Tsui, B. M. W. 1990. SPECT Dual-Energy-Window Compton correction: Scatter multiplier required for quantification. J. Nucl. Med. 31, 90-98.

[32] Lyra, M., Ploussi, A. and Georgantzoglou, A. 2011. MATLAB as a tool in Nuclear Medicine Image Processing, MATLAB - A Ubiquitous tool for the practical engineer, 477-500.

[33] Naum, A., Ursaru, M., Manea, P., Stefanescu, C., Negru, D. and Jari, I. 2017. Image reconstruction in cardiac SPECT image processing. Rev. Med. Chir. Soc. Med. Nat. 121, 689-94.

[34] Welvaert, M. and Rosseel, Y. 2013. On the definition of Signal-To-Noise Ratio and Contrast-To-Noise Ratio for fMRI data. PLoS ONE 8, e77089.

[35] Wieczorek, H. 2006. SPECT image quality and quantification. IEEE Nucl. Sci. Symp. Conf. Rec. 2854-58 